\title{
Manejo de derrame pericárdico: revisión sistemática de la literatura
}

\author{
Guillermo O. Trout ${ }^{a, *}$, Renata De La Hoz ${ }^{b}$, Lai M. Alfaro ${ }^{a}$, Angie P. Córdoba ${ }^{a}$ \\ y Gilberto A. Consuegra ${ }^{a}$ \\ a Facultad de Ciencias de la Salud, Universidad del Magdalena, Santa Marta, Colombia
b Facultad de Ciencias de la Salud Universidad del Magdalena, Escuela de Graduados, Universidad CES, Medellín, Colombia
}

Recibido el 4 de febrero de 2017; aceptado el 23 de octubre de 2017

Disponible en Internet el 14 de marzo de 2018

\section{PALABRAS CLAVE \\ Pericarditis; \\ Tratamiento; \\ Pericardio}

\begin{abstract}
Resumen
Objetivo: Revisar sistemáticamente la efectividad del tratamiento no quirúrgico para el manejo del derrame pericárdico moderado o severo.

Metodología: Se realizó una búsqueda sistemática desde febrero hasta junio de 2016 en las bases de datos Pub Med, SciELO y Lilacs, en inglés y español. Los términos de búsqueda utilizados fueron: Pericardial Effusion and Acute Pericarditis, Treatment, Therapy, Therapeutics, Management. Se eligieron artículos publicados entre 2011 y 2016 que abordaran el tratamiento del derrame pericárdico no quirúrgico y la pericarditis aguda. Se excluyeron artículos que evaluaran el manejo de taponamiento cardíaco, pericarditis constrictiva y derrame pericárdico por lesión miocárdica. La selección de artículos estuvo a cargo de dos evaluadores y, en caso de discrepancias, se consultó un tercer evaluador.

Resultados: Se identificaron un total de 2.998 referencias; posteriormente, se seleccionaron 138 artículos, de los cuales se evaluaron sus resúmenes. Se eligieron dos artículos para la lectura a texto completo, y se aplicaron a estos las guías Strobe, para estudios observacionales, y Consort, para ensayos clínicos aleatorizados. Se evidenció que la pericarditis recurrente ocurrió en 26 de los 120 pacientes en el grupo de colchicina y en 51 de 120 en el grupo placebo, RRR: 0,49 (IC = 0, 24-0, 65; $\mathrm{p}=0$ 0, 0009).

Conclusiones: la colchicina asociada a antiinflamatorios no esteroides evidenció ser efectiva en la pericarditis aguda idiopática y asimismo en sus recurrencias. Sin embargo, aún es necesario consultar más ensayos clínicos con mayor tamaño de muestra para determinar con exactitud la efectividad del tratamiento.

( ) 2018 Sociedad Colombiana de Cardiología y Cirugía Cardiovascular. Publicado por Elsevier España, S.L.U. Este es un artículo Open Access bajo la licencia CC BY-NC-ND (http:// creativecommons.org/licenses/by-nc-nd/4.0/).
\end{abstract}

\footnotetext{
* Autor para correspondencia.

Correo electrónico: troutguillermo@hotmail.com (G.O. Trout).
} 


\section{KEYWORDS}

Pericarditis;

Treatment;

Pericardial

\section{Management of pericardial effusion: systematic review of literature}

\begin{abstract}
Objective: To present a systematic review of the effectiveness of non-surgical treatment for the management of moderate or severe pericardial effusion.

Methodology: A systematic search was made from February to June 2016 in the databases of Pub Med, SciELO, and Lilacs, in English and Spanish. The search terms used were: Pericardial Effusion and Acute Pericarditis, Treatment, Therapy, Therapeutics, Management. Articles published between 2011 and 2016 that approached the non-surgical treatment of pericardial effusion and acute pericarditis were chosen. Articles that were excluded were those that evaluated cardiac tamponade, constrictive pericarditis, and pericardial effusion due to a myocardial lesion. The selection of the articles was the responsibility of two evaluators and, in case of discrepancies, a third evaluator was consulted.

Result: A total of 2,998 references where identified, from which 138 articles were later selected, and their Abstracts were evaluated. Two articles were chosen in order to read the full text, and the Strobe guidelines for observational studies and the Consort guidelines for randomised clinical trials were used. It was shown that recurrent pericarditis occurred in 26 of the 120 patients in the colchicine group, and in 51 of 120 in the placebo group, with a relative risk ratio (RRR): 0.49 (95\% Cl; 0.24- 0.65; $P=.0009)$.

Conclusions: Colchicine combined with non-steroidal anti-inflammatory drugs showed to be effective in acute idiopathic pericarditis, as well as in their recurrences. However, more clinical trials with a larger sample size need to be consulted in order to determine the effectiveness of the treatment with more accuracy.

(c) 2018 Sociedad Colombiana de Cardiología y Cirugía Cardiovascular. Published by Elsevier España, S.L.U. This is an open access article under the CC BY-NC-ND license (http:// creativecommons.org/licenses/by-nc-nd/4.0/).
\end{abstract}

\section{Introducción}

Las enfermedades del pericardio son relativamente comunes en la práctica clínica, por tanto son una causa frecuente de consulta e ingreso a los servicios de cardiología ${ }^{1}$. En cuanto al derrame pericárdico, es una patología ocasionada por diversos factores etiológicos, la cual se manifiesta con síntomas como disnea de esfuerzo que luego progresa a ortopnea, taquicardia, hipotensión, además de dolor torácico y sensación de plenitud, entre otros síntomas inflamatorios ${ }^{2-4}$. Su tratamiento debe estar enfocado a resolver la etiología y al manejo de los síntomas; no obstante, en situaciones en las que el diagnóstico es impreciso o es idiopático, y ante signos y elevación de marcadores inflamatorios, el manejo clínico debe ser igual al de la pericarditis ${ }^{2,3,5-11}$.

Teniendo en cuenta lo anterior el tratamiento de este tipo de casos se realiza con base en la administración de antiinflamatorios no esteroideos (AINE) debido a su efectividad, tolerancia y menores efectos adversos ${ }^{12,13}$. También, son usados fármacos como la aspirina, los diuréticos y la colchicina, el cual es un antiinflamatorio eficaz, que además, produce alivio del dolor; sin embargo, no debe usarse en pacientes embarazadas. Asimismo, se recomienda el uso de corticoides, especialmente cuando el derrame pericárdico se asocia a inflamación sistémica; no obstante, su uso está

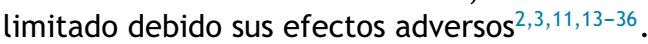

Aunque en la práctica clínica el tratamiento del derrame pericárdico no severo se hace con base en la administración de AINE; en la actualidad han surgido avances importantes en el tratamiento de estas enfermedades; un ejemplo de ello es la indicación de colchicina como fármaco de primera elección, el cual unido a la terapia convencional reduce las tasas de recurrencias ${ }^{3,16,32,37,38}$; generando así la necesidad de investigar la mejor estrategia terapéutica disponible con base en evidencia científica actualizada; pese a ello, aún persisten algunas limitaciones en cuanto a la difusión de estudios con rigurosidad adecuada y calidad metodológica, debido al acceso restringido de algunas bases de datos y la disponibilidad reducida de tiempo de los profesionales de la salud. En consecuencia, este artículo tiene como objetivo realizar una revisión de la literatura que incluya las estrategias terapéuticas previamente conocidas por la comunidad científica, y de esta manera identificar nuevas directrices en cuanto a su uso consignado en evidencia científica actualizada; asimismo, se pretende con su realización conocer la disponibilidad de nuevas pautas terapéuticas; y a partir de ello suministrar una valiosa fuente de información actualizada y resumida de estas.

\section{Metodología}

Se realizó una revisión sistemática de la literatura de acuerdo con las pautas consignadas en la guía PRISMA, siguiendo las etapas de identificación, cribado, elegibilidad e inclusión expuestas en la misma ${ }^{39,40}$. Se llevó a cabo una búsqueda sistemática desde febrero hasta junio del 2016 de artículos publicados en inglés y español en las bases de datos Pub Med, SciELO y Lilacs. Los términos empleados en la búsqueda se encuentran en la tabla 1. Se cribaron los artículos identificados, eliminando los duplicados y según el título, y 
Tabla 1 Términos y estrategia de búsqueda aplicados en la revisión sistemática

\begin{tabular}{l}
\hline Términos utilizados en la búsqueda \\
\#1 Pericardial effusion (MeSH) \\
\#2 Acute pericarditis \\
\#3 Treatment \\
\#4 Therapy \\
\#5 Therapeutics (MeSH) \\
\#6 Management (MeSH) \\
Estrategia de búsqueda \\
\#1 AND \#3 \\
\#1 AND \#4 \\
\#1 AND \#5 \\
\#1 AND \#6 \\
\#2 AND \#3 \\
\#2 AND \#4 \\
\#2 AND \#5 \\
\#2 AND \#6
\end{tabular}

con la lectura de los resúmenes de los mismos, teniendo en cuenta los siguientes criterios de inclusión: artículos publicados entre 2011 y 2016, que abordaran el tratamiento farmacológico de derrame pericárdico y pericarditis aguda, que hubieran sido realizados en seres humanos y en pacientes adultos.

Se aplicaron como criterios de exclusión artículos que evaluaran el manejo de taponamiento cardíaco, pericarditis constrictiva y derrame pericárdico por lesión miocárdica.

La búsqueda y la selección de artículos, con base en los criterios de inclusión y exclusión, estuvo a cargo de dos evaluadores independientes, y en caso de discrepancias se consultó un tercer evaluador.

Adicionalmente, se aplicaron las guías Strobe, para estudios observacionales, y Consort, para ensayos clínicos, a los estudios seleccionados de manera preliminar, según los criterios ya establecidos, con la finalidad de proporcionar el mayor nivel de evidencia a partir de la selección de estudios con un adecuado diseño metodológico. La metodología utilizada fue evaluada y replicada con el seguimiento del protocolo (fig. 1).

\section{Resultados}

A partir de la estrategia de búsqueda, se identificaron un total de 2.998 referencias en las bases de datos consultadas. Luego de eliminar los duplicados y los eliminados por

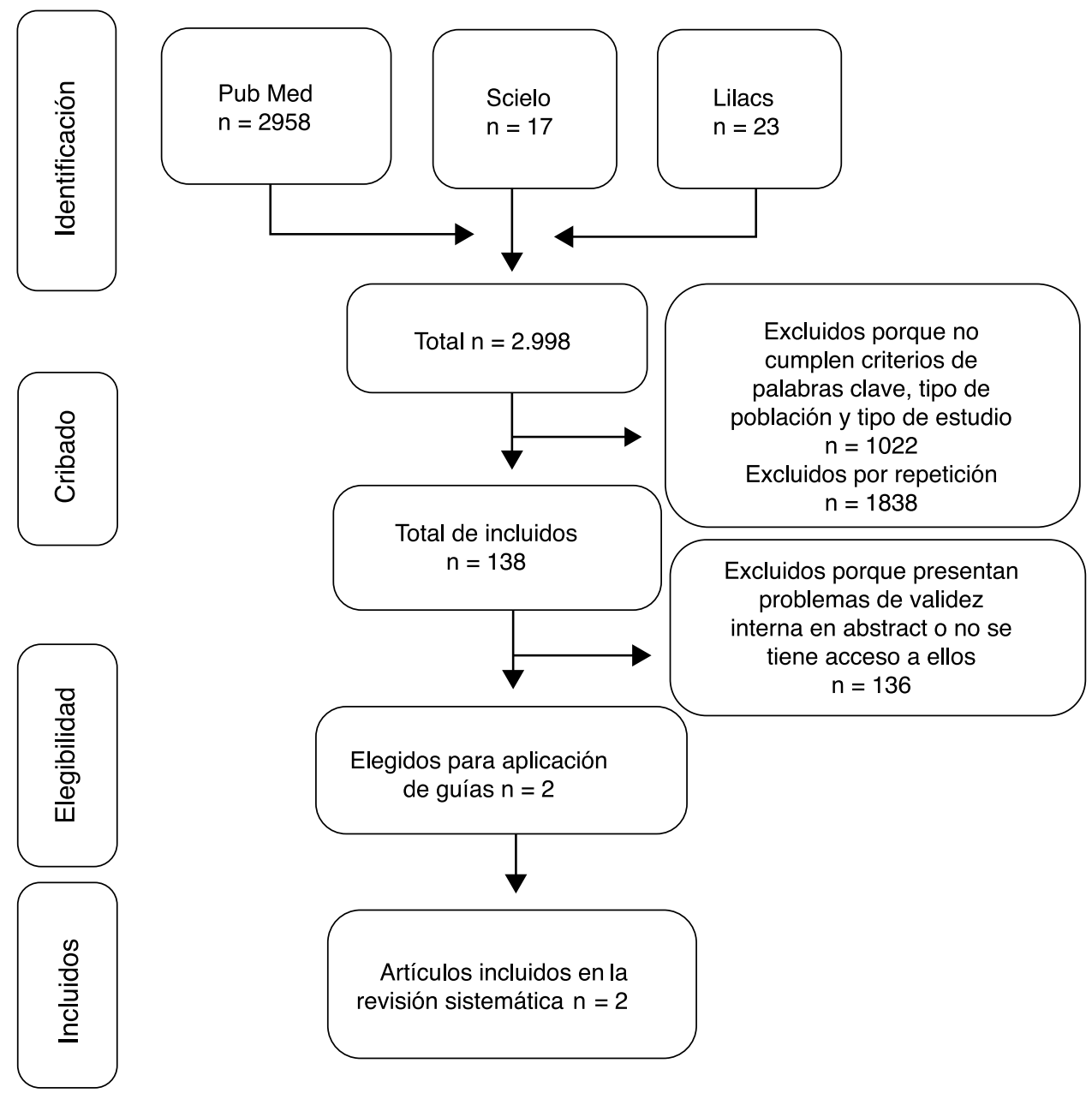

Figura 1 Flujograma del proceso de selección de los estudios. 
título, se revisaron 138 artículos para su evaluación por abstract. Posteriormente, se eligieron diez artículos para la lectura a texto completo y la posterior aplicación de las guías Consort y Strobe, para ensayos clínicos aleatorizados y estudios observacionales respectivamente, de los cuales solo dos superaron la rigurosidad metodológica requerida por las guías mencionadas.

Dentro de estos dos artículos se encuentra el publicado por Adler en 2013, en el cual se observó que la pericarditis recurrente o incesante ocurrió en el $16,7 \%$ de los pacientes en el grupo de colchicina y el $37,5 \%$ en el grupo de placebo, reducción del riesgo relativo (RRR) en el grupo colchicina, 0,56; con una confianza del 95\% [intervalo de confianza $(I C)=0,30-0,72 ; p<0,001)]$. Estos resultados fueron similares independientemente de la terapia concomitante (ibuprofeno o aspirina) usada durante el seguimiento. Asimismo, la colchicina redujo la frecuencia en la persistencia de los síntomas a las 72 horas $(19,2 \%$ frente a placebo $40,0 \% ; p=0,001), y$ adicionalmente prolongó el tiempo hasta la primera recurrencia (24,7 semanas frente a 17,7 semanas; $p<0,001)$. En cuanto a los valores globales de los efectos adversos estos fueron similares en ambos grupos de estudios $^{37}$ (tabla 3).

En cuanto al segundo artículo seleccionado, publicado por Adler en 2014, se evidenció que la pericarditis recurrente ocurrió en 26 de los 120 pacientes en el grupo de colchicina y en 51 de 120 en el grupo placebo, RRR 0, 49; con una confianza del 95\% (IC 0,24-0,65; $\mathrm{p}=0,0009$; número necesario a tratar [NNT]5); los cuales fueron similares independientemente de la terapia concomitante utilizada. Adicionalmente, la colchicina redujo la frecuencia en la persistencia de los síntomas a las 72 horas $(19,2 \%$ frente a placebo 44,2\%; $p=0,0001$ ) y el número de recurrencias por pacientes $(0,28$ frente a placebo 0,$63 ; p=0,0004)$. Respecto a los efectos adversos observados, la intolerancia gastrointestinal fue el principal evento ocurrido, los cuales se hallaron en proporciones similares en cada grupo ${ }^{38}$ (tabla $3)$.

En cuanto al grupo de este ensayo clínico en el cual se utilizaron los corticoides como terapia concomitante adicional, se observó que la recurrencia a los 18 meses en pacientes tratados previamente con corticoides ocurrió en el $62,5 \%$ de 24 pacientes en el grupo placebo frente al 31,3\% de 16 en el grupo colchicina $(p=0,10)$. Para los pacientes tratados con prednisolona durante el seguimiento, la recurrencia a los 18 meses ocurrió en el $52,2 \%$ de 23 pacientes frente al $36,8 \%$ de $19(p=0,50)$. Finalmente, es necesario aclarar que el efecto significativo de la colchicina en la pericarditis idiopática (42,2\% de 102 en comparación con placebo $18,8 \%$ de 96 ; $\mathrm{p}=0,0006)$, no se observó en aquellos pacientes con pericarditis no idiopática $(44,4 \%$ de 18 frente a placebo $33,3 \%$ de $24 ; p=0,68)^{38}$ (tabla 2).

\section{Discusión}

En ausencia de un diagnóstico establecido, el uso de antiinflamatorios no esteroideos está recomendado para el manejo del derrame pericárdico no severo y sus recurrencias $^{18}$. Sin embargo, desde hace algún tiempo existen otras recomendaciones que incluyen combinaciones entre fármacos antiinflamatorios, además de nuevas opciones terapéuticas.

Por otra parte, es preciso aclarar que dentro del proceso de realización de este artículo se encontraron resultados similares a las recomendaciones hechas por la "Guía europea para enfermedades del pericardio 2015" en diversas revisiones narrativas halladas durante la búsqueda; sin embargo, se destaca que dentro de ésta sólo diez artículos cumplieron los criterios para ser seleccionados preliminarmente, de los cuales seis fueron ensayos clínicos aleatorizados que abordaron la eficacia de la colchicina para la prevención de las recurrencias posterior a pericardiotomía ${ }^{41-44}$, y un séptimo subestudio de un ensayo clínico aleatorizado publicado por Bunge en 2014 en el cual se abordaba el manejo del síndrome postpericardiotomía con dexametasona, obteniendo como resultado la pobre efectividad de este tratamiento ${ }^{45}$. Los tres estudios restantes fueron observacionales de cohorte, de los cuales sólo uno abordaba el tema del tratamiento del manejo pericárdico; sin embargo, en este se tuvieron en cuenta pacientes con diagnóstico de VIH en tratamiento con terapia antirretroviral $^{46}$. Una limitante en esta revisión es que solo dos artículos cumplieron el análisis realizado por los evaluadores al aplicar las guías Consort y Strobe durante su lectura completa, los cuales de forma coincidencial evaluaban la efectividad de la colchicina en este tipo de patologías.

En cuanto a los hallazgos hechos durante la revisión de los dos artículos seleccionados por su rigurosidad metodológica se destaca que la colchicina añadida a la terapia antiinflamatoria convencional puede reducir el tiempo entre la aparición de nuevas recurrencias de la pericarditis aguda. Pese a la variedad del tratamiento concomitante utilizado, entre los cuales se destacan el ibuprofeno, la indometacina y la aspirina, no se hallaron diferencias significativas entre estos en lo que concierne a la efectividad observada ${ }^{37,38}$.

Durante la revisión se observaron semejanzas en ambos estudios en cuanto a la metodología aplicada, lo que posiblemente incidió en la similitud de los resultados encontrados en ambos ensayos clínicos; dentro de éstos se destacan, el porcentaje de recurrencias, la frecuencia de los síntomas a las 72 horas, los tipos de eventos adversos encontrados, y por último la frecuencia de los mismos. No obstante, se encontraron algunas variaciones, la principal tuvo relación con el tiempo de administración de la dosis dada, debido a que en el estudio de Adler de 2014, esta fue administrada durante 6 meses sin una dosis de carga, mientras que en la publicación de Adler de 2013 se administró durante 3 meses igualmente sin una dosis de $\operatorname{carga}^{37,38}$; sin embargo esto no tuvo gran relevancia.

Por otra parte, estos estudios no han sido los únicos en obtener resultados similares en cuanto a la efectividad de la colchicina combinada con la terapia convencional para tratar la pericarditis recurrente, debido a que en otros estudios como el realizado por Trinchero en 2005, también se encontró que la colchicina combinada con la terapia convencional disminuyó el porcentaje de recurrencias luego de un primer episodio de pericarditis aguda en comparación con la administración única de aspirina $(10,7$ y $32,3 \%$, respectivamente; $p=0,004$; con una confianza del $95 \%$ IC $=3,1$ a 10,0; NNT = 5). Asimismo, otro estudio similar a este, realizado por el mismo autor en septiembre del mismo año, 
Tabla 2 Características de la intervención administrada y porcentaje de recurrencia de los estudios evaluados

\begin{tabular}{|c|c|c|c|c|c|c|c|}
\hline Autor y año & Tipo de estudio & $\begin{array}{l}\text { Tamaño de } \\
\text { muestra }\end{array}$ & $\begin{array}{l}\text { Tiempo de } \\
\text { seguimiento }\end{array}$ & Intervención & $\begin{array}{l}\text { Clase y nivel } \\
\text { pericarditis } \\
\text { aguda }\end{array}$ & $\begin{array}{l}\text { Clase y nivel } \\
\text { de derrame } \\
\text { pericárdico }\end{array}$ & $\begin{array}{l}\text { Resultados } \\
\text { principales }\end{array}$ \\
\hline $\begin{array}{l}\text { Adler Y. } \\
2013^{37}\end{array}$ & $\begin{array}{l}\text { Ensayo } \\
\text { multicéntrico, } \\
\text { aleatorizado, } \\
\text { doble ciego y } \\
\text { placebo } \\
\text { controlado }\end{array}$ & $\begin{array}{l}\text { Muestra total= } \\
240, \text { grupo de } \\
\text { placebo } 120, \\
\text { grupo de } \\
\text { colchicina } 120\end{array}$ & 18 meses & $\begin{array}{l}\text { Colchicina de } \\
0,5 \mathrm{mg} \text { cada } 24 \\
\text { h en pacientes } \\
<70 \mathrm{~kg} \text { y } 0, \\
5 \mathrm{mg} \text { cada } 12 \mathrm{~h} \\
\text { en pacientes } \\
>70 \mathrm{~kg} \text { por } 3 \\
\text { meses, más } \\
\text { terapia con } \\
\text { Aspirina o } \\
\text { Ibuprofreno }\end{array}$ & $1 \mathrm{~A}(4)$ & $1 \mathrm{C}(4)$ & $\begin{array}{l}\text { Se observó que } \\
\text { la pericarditis } \\
\text { recurrente o } \\
\text { incesante } \\
\text { ocurrió en } 20 \\
\text { pacientes } \\
(16,7 \%) \text { en el } \\
\text { grupo de } \\
\text { colchicina y en } \\
45 \text { pacientes } \\
\text { ( } 37,5 \% \text { ) en el } \\
\text { grupo de } \\
\text { placebo (RRR } \\
56 \% \text {; RR } 0,44 ; \\
\text { IC 95\% 0,30 - } \\
0,72 ; p<0,001 \text {; } \\
\text { NNT 4) }\end{array}$ \\
\hline Adler Y, $2014^{38}$ & $\begin{array}{l}\text { Ensayo } \\
\text { multicéntrico, } \\
\text { aleatorizado, } \\
\text { doble ciego y } \\
\text { placebo } \\
\text { controlado }\end{array}$ & $\begin{array}{l}\text { Muestra total= } \\
240, \text { grupo de } \\
\text { placebo } \\
120 \text {,grupo de } \\
\text { colchicina } 120\end{array}$ & 20 meses & $\begin{array}{l}\text { Colchicina de } \\
0,5 \mathrm{mg} \text { cada } 24 \\
\text { h en pacientes } \\
<70 \mathrm{~kg} \text { o } 0,5 \mathrm{mg} \\
\text { cada } 12 \mathrm{~h} \text { en } \\
\text { pacientes } \\
>70 \mathrm{~kg} \text {, por } 6 \\
\text { meses, más } \\
\text { terapia con } \\
\text { Aspirina de } \\
800 \mathrm{mg}, \\
\text { Ibuprofeno de } \\
600 \text { mg o } \\
\text { Indometacina } \\
\text { de } 50 \text { mg, y en } \\
\text { algunos } \\
\text { pacientes } \\
\text { recibieron } \\
\text { prednisolona } \\
0,2-0,5 \text { mg / kg } \\
\text { por día para } 4 \\
\text { semanas }\end{array}$ & , & $1 \mathrm{C}(4)$ & $\begin{array}{l}\text { Se evidenció } \\
\text { que la } \\
\text { pericarditis } \\
\text { recurrente } \\
\text { ocurrió en } 26 \\
\text { pacientes ( } 21 \text {, } \\
6 \% \text { ) en el grupo } \\
\text { de colchicina y } \\
51 \text { en el grupo } \\
\text { placebo (RRR } \\
51 \% \text {; RR } 0,49 ; \\
\text { IC } 95 \% 0,24- \\
0,65 ; p=0,0009 \text {; } \\
\text { NNT 5) }\end{array}$ \\
\hline
\end{tabular}

*Clase (clase de recomendación); Nivel (nivel de evidencia), mg (miligramos), h (horas) kg (kilogramos), RRR (reducción de riesgo relativo) RR (riesgo relativo), IC (intervalo de confianza), p de significancia, NNT (números necesarios a tratar).

Tabla 3 Efectos adversos presentados en los estudios seleccionados según la intervención realizada

\begin{tabular}{|c|c|c|c|c|}
\hline \multirow[b]{2}{*}{$\begin{array}{l}\text { Principales efectos } \\
\text { adversos durante los } \\
\text { estudios }\end{array}$} & \multicolumn{2}{|c|}{ Adler Y, $2013^{37}$} & \multicolumn{2}{|c|}{ Adler Y, $2014^{38}$} \\
\hline & $\begin{array}{l}\text { Grupo placebo } \\
(n=120)\end{array}$ & $\begin{array}{l}\text { Grupo } \\
\text { colchicina } \\
(n=120)\end{array}$ & $\begin{array}{l}\text { Grupo placebo } \\
(n=120)\end{array}$ & $\begin{array}{l}\text { Grupo } \\
\text { colchicina } \\
(n=120)\end{array}$ \\
\hline $\begin{array}{l}\text { Intolerancia } \\
\text { gastrointestinal }\end{array}$ & $(8,3 \%)$ & $(9,2 \%)$ & $(7,5 \%)$ & $(7,5 \%)$ \\
\hline Hepatotoxicidad & $(0,8 \%)$ & $(1,7 \%)$ & $(0,8 \%)$ & $(2,5 \%)$ \\
\hline Miotoxicidad & $(0,0 \%)$ & $(0,0 \%)$ & $(0,0 \%)$ & $(0,8 \%)$ \\
\hline Alopecia & $(0,8 \%)$ & $(0,8 \%)$ & $(0,0 \%)$ & $(0,8 \%)$ \\
\hline
\end{tabular}

* Se incluyen náuseas, vómitos, dolor abdominal, cólicos y diarrea. 
obtuvo una reducción en el porcentaje de recurrencias en el grupo de colchicina más terapia convencional superior al grupo en el cual solo se evaluó esta última $(24,0 \%$ frente $50,6 \%$ respectivamente; $p=4,0$; con una confianza del $95 \%$ $\mathrm{IC}=2,5-7,1)^{16,32}$.

Aunque, la colchicina usualmente se recomienda como primera línea de tratamiento en asociación con los AINE, administrada en una dosis dependiente del peso, para el manejo de la pericarditis aguda y el derrame pericárdico ${ }^{47-50}$, no se encontró un efecto significativo en la reducción de la recurrencia en pericarditis no idiopática [ocho $(44,4 \%=\text { de } 18 \text { versus ocho }(33 \cdot 3 \%) \text { de } 24 ; p=0,68]^{38}$.

Pese a que la búsqueda inicialmente arrojó un gran número de resultados, es relevante afirmar que la mayoría se trataba de reportes de casos y revisiones narrativas basadas en las guías europeas para enfermedades del pericardio, lo cual redujo de manera importante el número de resultados posibles basados en evidencia científica de calidad, constituyendo así la primera limitante. Por otra parte, los ensayos clínicos aleatorizados multicéntricos hallados abordaban únicamente la colchicina como tratamiento del derrame pericárdico en pacientes sometidos a procedimientos quirúrgicos basados en la efectividad que esta tiene en el manejo de la enfermedad en condiciones de no severidad. Una tercera limitación es el tamaño reducido de la muestra hallado en los artículos revisados, lo cual motiva la consulta de más ensayos clínicos con un mayor tamaño de muestra para determinar con más exactitud la efectividad del tratamiento y sus efectos adversos.

Finalmente, se recomienda continuar con las directrices aprobadas por la guía europea haciendo énfasis en los resultados obtenidos con la colchicina durante la presente revisión; además de resaltar la importancia de la rigurosidad metodológica de los estudios realizados para una adecuada reproducción de sus hallazgos a la comunidad científica.

\section{Conclusiones}

La colchicina asociada a AINE demostró ser efectiva no solo en pericarditis aguda idiopática no recurrente, sino como un posible tratamiento en las recurrencias de esta enfermedad. Sin embargo, aún no hay resultados concluyentes acerca de su efectividad en la pericarditis aguda no idiopática. Además, se identificó como un medicamento seguro cuando se administra en la dosis equivalente al peso del paciente. En lo que respecta a la duración del tratamiento, en ambos estudios mostró buenos resultados, independiente de las diferencias que se tuvieron en los ensayos observados en cuanto al tiempo de administración ${ }^{37,38}$.

\section{Financiación}

Ninguna.

\section{Conflictos de interés}

Ninguno.

\section{Bibliografía}

1. Marín JE, Duque M, Uribe W, Medina E. Pericardial disease and myocarditis: management guide. Rev Colomb Cardiol. 2005;11:319-32.

2. Azam S, Hoit BD. Treatment of pericardial disease. Cardiovasc Ther. 2011;29:308-14.

3. Guindo J. Comentarios a la guía ESC 2015 sobre el diagnóstico y tratamiento de las enfermedades del pericardio, Un informe del Grupo de Trabajo del Comité de Guías de la Sociedad Española de Cardiología. Rev Esp Cardiol. 2015;68:1068-74.

4. Imazio M. Pericardial involvement in systemic inflammatory diseases. Heart. 2011;97:1882-92.

5. Imazio M, Mayosi BM, Brucato A, Adler Y. Pericardial effusion triage. Int J Cardiol. 2010;145:403-4.

6. Linhart A. Summary of 2015 ESC guidelines for the diagnosis and management of pericardial diseases Prepared by the Czech society of Cardiology. Coret Vasa. 2016;58:106-81.

7. Imazio M, Mayosi BM, Brucato A, Markel G, Trinchero R, Spodick D, Adler Y. Triage and management of pericardial effusion. J Cardiovasc Med (Hagerstown, Md.). 2010;11:928-35.

8. Imazio M, Brucato A, Mayosi BM, Derosa FG, Lestuzzi C, Macor $A$, et al. Medical therapy of pericardial diseases: part II: Noninfectious pericarditis, pericardial effusion and constrictive pericarditis. J Cardiovasc Med Hagerstown Md. 2010;11:785-94.

9. Imazio M, Trinchero R. Clinical management of acute pericardial disease: a review of results and outcomes. Ital Heart J Off J Ital Fed Cardiol. 2004;5:803-17.

10. Guidelines on the Diagnosis and Management of Pericardial Diseases Executive Summary The Task Force on the Diagnosis and Management of Pericardial Diseases of the European Society of Cardiology. Eur Heart J. 2004;25(7):587-610.

11. Maisch B, Ristic AD. Practical aspects of the management of pericardial disease. Heart. 2003;89:1096-103.

12. Adler Y, Guindo J, Finkelstein Y, Khouri A, Assali A, Bayes-Genis $A$, et al. Colchicine for large pericardial effusion. Clin Cardiol. 1998;21:143-4.

13. Guindo Soldevila J. Unidades de cuidados intensivos cardiológicos más allá del infarto. Madrid: Sociedad Española de Cardiología; 2009.

14. Imazio M, Spodick DH, Brucato A, Trinchero R, Adler Y. Controversial issues in the management of pericardial diseases. Circulation. 2010;121:916-28.

15. Villamañán $E$, Larrubia $Y$, Ruano $M$, Colquicina:. ¿qué hay de nuevo, viejo? Med Clin. 2012;139:295-9 [Internet]. [Acceso 25 Aug 2016]. Disponible en: http://www.elsevier.es/ es-revista-medicina-clinica-2-articulo-colquicina-que-haynuevo-viejo-S0025775312001625? redirectNew=true

16. Imazio M, Bobbio M, Cecchi E, Demarie D, Pomari F, Moratti M, et al. Colchicine as first-choice therapy for recurrent pericarditis: results of the CORE (COlchicine for REcurrent pericarditis) trial. Arch Intern Med. 2005;165:1987-91.

17. Klein AL, Abbara S, Agler DA, Appleton CP, Asher CR, Hoit $B$, et al. American Society of Echocardiography Clinical Recommendations for Multimodality Cardiovascular Imaging of Patients with Pericardial Disease. J Am Soc Echocardiogr. 2013;26:965-1012e15.

18. Imazio M, Adler Y. Management of pericardial effusion. Eur Heart J. 2013;34:1186-97.

19. Sagristà-Sauleda J, Mercé AS, Soler-Soler J. Diagnosis and management of pericardial effusion. World J Cardiol. 2011;3:135-43.

20. Adlam DFC. Pericardial disease. In: Cardiology Medicine International. Prendergast BD: Oxford University press; 2010.

21. Meurin P, Lelay-Kubas S, Pierre B, Pereira H, Pavy B, Iliou $M C$, et al. Colchicine for post-operative pericardial effusion, Preliminary Results of the POPE-2 Study. J Am Coll Cardiol. 2015;66:1198-9. 
22. Imazio M, Demichelis B, Parrini I, Giuggia M, Cecchi E, Gaschino G, et al. Day-hospital treatment of acute pericarditis: A management program for outpatient therapy. J Am Coll Cardiol. 2004;43:1042-6.

23. Little WC, Freeman GL. Pericardial disease. Circulation. 2006;113:1622-32.

24. Lotrionte M, Biondi-Zoccai G, Imazio M, Castagno D, Moretti C, Abbate A, et al. International collaborative systematic review of controlled clinical trials on pharmacologic treatments for acute pericarditis and its recurrences. Am Heart J. 2010;160:662-70.

25. Kloos JA. Characteristics, complications, and treatment of acute Pericarditis - critical care nursing clinics. Critical Care Nursing Clinics. 2015;27:483-97.

26. Hoit Bd. Cardiovascular Therapeutics. $3^{\text {rd }}$. Ed. Elsevier; 2007. p. 787-97. Chapter 45 - treatment of Pericardial disease.

27. Khandaker MH, Espinosa RE, Nishimura RA, Sinak LJ, Hayes SN, Melduni RM, et al. Pericardial disease: diagnosis and management. Mayo Clin Proc. 2010;85:572-93.

28. Shammas NW, Padaria RF, Coyne EP. Pericarditis, myocarditis, and other cardiomyopathies. Prim Care Clin Off Pract. 2013;40:213-36.

29. Foerg FGZ. Pericarditis. Hospital Medicine Clinics. 2015:205-15.

30. Imazio M, Brucato A, Trinchero R, Spodick D, Adler Y. Colchicine for pericarditis: hype or hope? Eur Heart J. 2009;30:532-9.

31. Imazio M, Brucato A, Cumetti D, Brambilla G, Demichelis B, Ferro S, et al. Corticosteroids for recurrent pericarditis: high versus low doses: a nonrandomized observation. Circulation. 2008;118:667-71.

32. Imazio M, Bobbio M, Cecchi E, Demarie D, Demichelis B, Pomari $F$, et al. Colchicine in addition to conventional therapy for acute pericarditis: results of the COlchicine for acute PEricarditis (COPE) trial. Circulation. 2005;112:2012-6.

33. Freixa X. Evaluación, manejo y tratamiento de las pericarditis y miocarditis agudas en urgencias. Emerg Rev Soc Esp Med Urgenc Emerg. 2010;22:301-6.

34. Imazio M. Tratamiento de la pericarditis recurrente. Rev Esp Cardiol. 2014;67:345-8.

35. Román FRJ, De la Cruz Aviles L, Hernández RV, Mena FR. Guía de práctica clínica para el tratamiento de la pericarditis aguda. Medisur. 2009; 7:217-21.

36. Guindo J, Sagristá J. Derrame pericárdico. Neoplasia. 2007;13:15.

37. Imazio M, Brucato A, Cemin R, Ferrua S, Maggiolini S, Beqaraj $\mathrm{F}$, et al. A randomized trial of colchicine for acute pericarditis. N Engl J Med. 2013;369:1522-8.

38. Imazio M, Belli R, Brucato A, Cemin R, Ferrua S, Beqaraj $F$, et al. Efficacy and safety of colchicine for treatment of multiple recurrences of pericarditis (CORP-2): a multicentre, double-blind, placebo-controlled, randomised trial. The Lancet. 2014;383:2232-7.

39. Urrútia G, Bonfill X. Declaración PRISMA: una propuesta para mejorar la publicación de revisiones sistemáticas y metaanálisis. Med Clin (Barc). 2010;135:507-11.

40. Hutton B, Catalá-López F, Moher D. La extensión de la declaración PRISMA para revisiones sistemáticas que incorporan metaanálisis en red: PRISMA-NMA. Med Clin (Barc). 2016;147:262-6.

41. Imazio M, Belli R, Brucato A, Ferrazzi P, Patrini D, Martinelli $\mathrm{L}$, et al. Rationale and design of the Colchicine for Prevention of the Post-pericardiotomy Syndrome and Post-operative Atrial Fibrillation (COPPS-2 trial): a randomized, placebo-controlled, multicenter study on the use of colchicine for the primary prevention of th. Am Heart J. 2013;166:13-9.

42. Meurin P, Lelay-Kubas S, Pierre B, Pereira H, Pavy B, Iliou MC, et al. Colchicine for postoperative pericardial effusion: a multicentre, double-blind, randomized controlled trial. Heart. 2015;101:1711-6.

43. Imazio M, Brucato A, Rovere ME, Gandino A, Cemin R, Ferrua S, et al. Colchicine prevents early postoperative pericardial and pleural effusions. Am Heart J. 2011;162:527-32.

44. Imazio M, Brucato A, Ferrazzi P, Pullara A, Adler Y, Barosi A, et al. For the COPPS-2 Investigators Colchicine for Prevention of Postpericardiotomy Syndrome and Postoperative Atrial Fibrillation The COPPS-2 Randomized Clinical Trial. JAMA. 2014;312:1016-23.

45. Bunge JJ, van Osch D, Dieleman JM, Jacob KA, Kluin J, van Dijk $D$, et al., for the Dexamethasone for Cardiac Surgery (DECS) Study Group. Dexamethasone for the prevention of postpericardiotomy syndrome: A DExamethasone for Cardiac Surgery substudy. Am Heart J. 2014;168:126-31.

46. Lind A, Reinsch N, Neuhaus K. Pericardial effusion of HIVinfected patients? Results of a prospective multicenter cohort study in the era of antiretroviral therapy. Eur J Med Res. 2011;16:480-3.

47. Imazio M, Brucato A, Belli R, Forno D, Ferro S, Trinchero R, et al. Colchicine for the prevention of pericarditis: what we know and what we do not know in 2014 - systematic review and metaanalysis. J Cardiovasc Med Hagerstown Md. 2014;15:840-6.

48. Alabed S, Cabello JB, Irving GJ, Qintar M, Burls A. Colchicine for pericarditis. Cochrane Database Syst Rev. 2014:CD010652.

49. Soler-Soler J, Sagristà-Sauleda J, Permanyer-Miralda G. Relapsing pericarditis. Heart. 2004;90:1364-8.

50. Schwier NC, Coons JC, Rao SK. Pharmacotherapy update of acute idiopathic pericarditis. Pharmacotherapy. 2015;35: 99-111. 